\title{
A Rapid Prototyping Tool for Interactive Device Development
}

\author{
Mark Merlo $^{1,2}$ and Mark Bachman ${ }^{1}$ \\ ${ }^{1}$ University of California, Irvine, The Henry Samueli School of Engineering, University of \\ California, Irvine, CA 92697-2700, USA \\ ${ }^{2}$ Wildflower Technologies, Inc. 1300 S. Bristol St., Santa Ana, CA. 93704 \\ mwmerlo@wildflowertechnologies.com, mbachman@uci.edu
}

\begin{abstract}
Designers need rapid prototyping tools that are embeddable, easily configured and can control a large range of accessories. Current prototyping tools fall short on these requirements by requiring one or more of the following: a tether to a computer, textual programming, and/or limited accessory control. To overcome the limitations of current tools, we have developed Buttercup, a standalone embedded sensor/effector controller that provides a high degree of customization for rapid prototyping interactive devices. The keys to the implementation of Buttercup are its hardware and firmware architecture. By building a system focused on sensor and effector control, the hardware can be small and inexpensive. The firmware utilizes a unique mapping system that lends itself to robust control over its accessories while allowing intuitive configuration by the user through a graphical user interface.
\end{abstract}

Keywords: Embedded controller, rapid prototyping, graphical programming, physical interactive system, effector controller, sensor controller.

\section{Introduction}

Designers are increasingly adding electronics and computers to products to increase their functionality and interactivity [1]. Rapid prototyping and the iterative design process are critical to the development of new products [2]. It is typically difficult or prohibitively expensive to incorporate form and function into a single prototype and, therefore, form and function are routinely divided during the development and prototyping of new devices. This difficulty is compounded by the time and cost that it takes to add or change features of a functional prototype [3]. This leads to fewer functional prototypes during the prototyping phase and the design process suffers from fewer iterations.

Tools that enable functional prototypes are of great value to the designer. From the designer's perspective, the ideal prototyping tool would be embeddable and small, be easy to configure with no textual programming, and offer control over a large range of sensors and effectors (i.e. devices that modify the environment or provide stimulus to humans; examples include lights, sounds, vibrators, motors, and heaters). Embeddability and large range of accessories are important to make the tool as universal as possible. 
Ease of configuration without programming is critical since designers generally do not have a programming background [3].

Other groups have identified these needs and have developed systems to aid the designer in the prototyping process. Despite these efforts, with the exception of the Buttercup platform presented here, no prototyping tool satisfies the three needs listed above (i.e., embeddable, simple configuration and control of large range of sensors and effectors).

Many prototyping tools have focused on the information appliance [4-6] due to its popularity and ubiquity in today's culture. An information appliance is a computer designed to perform a specific function; examples include mobile phones, global positioning systems (GPS) devices and MP3 players [7]. Even though it is not explicitly stated in the definition of information appliances, these devices almost always interact with the user through a visual display, audio or both. Since both visual displays and audio require high bandwidth and fast computing power, almost all prototyping tools use a traditional PC to control these outputs and thereby tether, either wired or wirelessly, the prototyping tools to a computer. This tether usually prevents the device from being embedded or limits the range the device is functional. Some examples of these prototyping tools include d.tools [5], Thumbtacks [8], and DisplayObjects [6].

Phidgets [9] has the ability to control many types of sensors and effectors, but still requires a tether to a PC. The Arduino and iStuff Mobile [10] can work independent of a computer and lend themselves nicely to being embedded. The Arduino can also control many sensor and effector types, but requires textual programming to operate and configure (a severe drawback to less program-savvy designers). iStuff Mobile suffers from lack of effector support and though it can be visually programmed, the type of visual programming is aimed towards programmers.

To overcome the shortcomings of current rapid prototyping tools, we have developed the Buttercup platform, an embeddable sensor/effector controller that can be intuitively configured visually.

\section{Platform Overview}

The Buttercup is a standalone embedded sensor/effector controller that provides a high degree of customization for rapid prototyping interactive systems. Fig. 1 shows the Buttercup controller along with some sensors and effectors that are compatible with the system. In its most basic operation, sensor data is read by the Buttercup controller which uses a user-defined response curve to generate control signals for an effector.

The block diagram of a generic system setup in Fig. 2 shows the basic system components and typical data flow. The four basic subsystems of this platform are: the Buttercup controller, a sensor, an effector and power. There is also an optional connection to a computer via USB that is used to configure the device using a graphical user interface (GUI) and to monitor its operating parameters in real-time. After the designer has configured the device, the parameters can be saved to nonvolatile memory and the Buttercup controller can be disconnected from the computer. When power is supplied to the Buttercup device (e.g. from batteries), the parameters that were saved are loaded and the system will operate independent of a computer. 


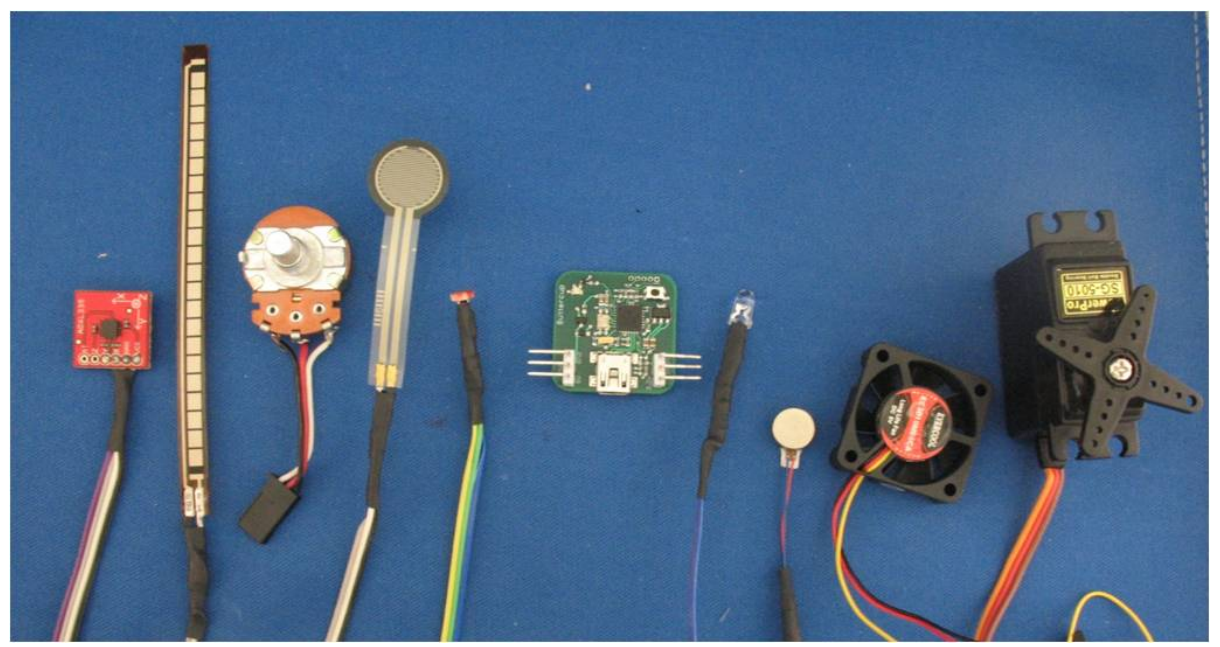

Fig. 1. Common components used in the Buttercup platform. From left to right: accelerometer, flex sensor, potentiometer, pressure sensor, light sensor, Buttercup controller (center), LED, vibrator, fan, servo-motor.

\section{Platform Details}

\subsection{Buttercup Controller}

The Buttercup controller is a $26 \mathrm{~mm} \times 26 \mathrm{~mm}$ device that is used to read sensor data and control an effector. The main hardware components of the Buttercup controller are a PIC18F24J50 microcontroller, a $5 \mathrm{~V}$ to $3.3 \mathrm{~V}$ voltage regulator, two crystals $(32.768 \mathrm{kHz}$ and $12 \mathrm{MHz}$ ), a mini-USB connector and headers for a sensor and an effector. The interfaces to the sensor and actuator operate on $5 \mathrm{~V}$ power and the microcontroller and all the supporting components operate on $3.3 \mathrm{~V}$ power. The analog-to-digital converter (ADC) on the microcontroller is 8-bits and therefore can record 256 discrete sensor values. During duty cycle control, the pulse width modulated (PWM) output of the microcontroller operates at $40 \mathrm{kHz}$ with a resolution of 8-bits, or 256 discrete duty cycles. This PWM frequency was selected to be above the upper threshold of human hearing $(\sim 20 \mathrm{kHz})$ and therefore limit any audible sound that might be generated when driving certain effectors. In servo mode, the PWM frequency is $50 \mathrm{~Hz}$ and the pulse width ranges from $1.2 \mathrm{~ms}$ to $1.8 \mathrm{~ms}(1.5 \mathrm{~ms}$ centered) at a resolution of $1 \mu \mathrm{s}$. This control signal is typical of most servo-motors.

\subsection{Sensor}

The three pin sensor connector from the Buttercup controller provides ground, $5 \mathrm{~V}$ power and analog signal in. The analog input signal should range between 0 and $5 \mathrm{~V}$. The sensor response is not required to be linear since the designer can correct for nonlinear sensors in the configuration of the Buttercup controller, but the sensor should have an impedance of $10 \mathrm{kOhms}$ or less to provide sufficient current to the $\mathrm{ADC}$ of the microcontroller. 


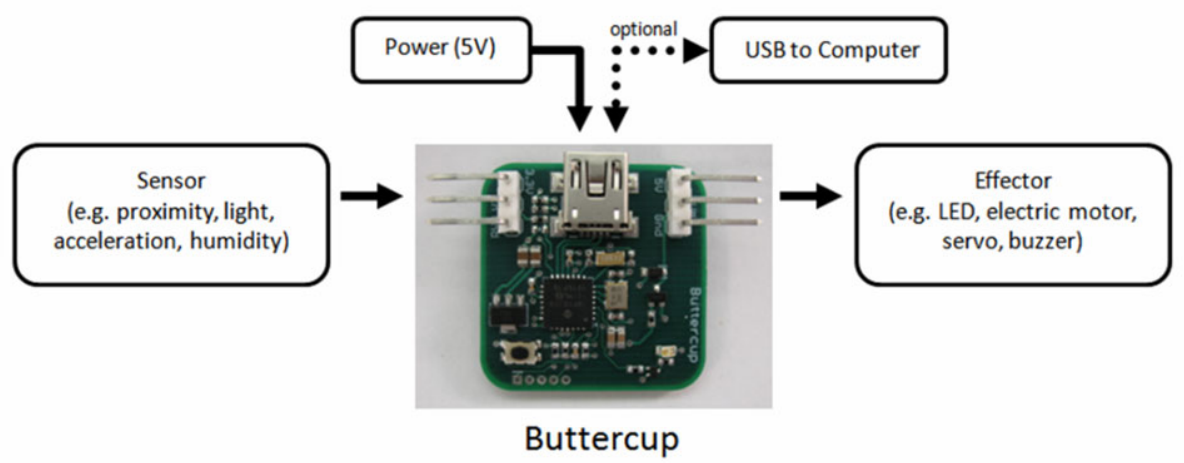

Fig. 2. Block diagram of a generic interactive system utilizing the Buttercup controller

\subsection{Effector}

The effector can be any device that is controllable by a PWM signal. This includes motors, LEDs, vibrators and speakers. The effector can also be controllable by pulse width duration, such as a servo-motor, instead of the typical duty cycle controlled devices mentioned above. The three pin effector connector from the Buttercup controller provides ground, $5 \mathrm{~V}$ power and PWM signal out.

\subsection{Power}

The operating supply power can range from $4.5 \mathrm{~V}$ to $5.5 \mathrm{~V}$. Power is provided to the Buttercup controller through the USB connector from either the $5 \mathrm{~V}$ USB bus power or from batteries during standalone operation. Power to the effector and the sensor are provided by their respective connectors on the Buttercup module.

\section{Platform Architecture}

There are three main elements to Buttercup's architecture that enable its functionality and versatility as shown in Fig. 3. The entire system update period is $1 \mathrm{~ms}$, which is

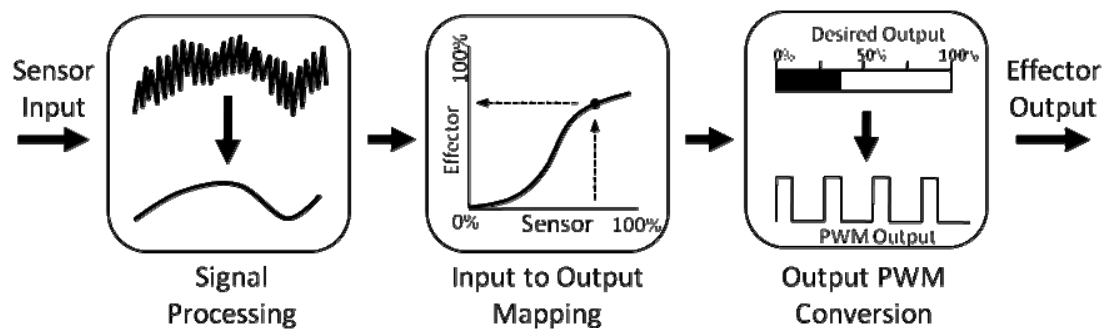

Fig. 3. Diagram of Buttercup architecture: Sensor data is processed from left to right to determine the proper effector control signal 
fast enough for any human interface device since the discrete steps are not perceivable and the system latency is not noticeable.

The first element, the signal processing step, reduces the noise of the analog sensor signal by oversampling the sensor data at $1 \mathrm{kHz}$. The data sampled is averaged over the system update period of $1 \mathrm{~ms}$, thereby averaging 10 readings for each update period. This signal processing reduces the noise from the sensor and eliminates most high frequency signals without affecting the response of the system.

The second element, sensor-to-effector mapping, enables the designer to arbitrarily control the effector's response to the sensor signal. By providing complete control over the sensor-to-effector responsive curve, the designer has the ability to fine-tune every aspect of the effector's response throughout the entire sensor range. This includes simple adjustments such as limiting the maximum or minimum effector output to more complex responses such as linearizing the sensor output or even reversing the effector response as the sensor signal increases (i.e., the effector goes from $0 \%$ to $100 \%$ back to $0 \%$ when the sensor signal goes from $0 \%$ to $100 \%$ ). This sensor-to-effector response is the same curve that is labeled C in Fig. 4.

In the firmware this response curve is stored as a 256 element lookup table, with the array index being the raw sensor value as determined by the signal processing step. A lookup table was selected over interpolation for several reasons. First, since the sensor data is only 8-bits and the PWM output resolution is 8-bits, a lookup is not prohibitively large, even for a microcontroller. Second, computation time is much smaller than other methods, such as interpolation, since the microcontroller does not have a floating point coprocessor. Finally, the lookup table gives designers complete control over the response curve and does not limit them to finite control of just a few points.

The third element, output PWM conversion, adjusts the output PWM signal to the effector based on the desired effector output. Depending on the effector, different PWM signals are needed to control it throughout its full range. For example, to turn an LED from $0 \%$ (fully off) to (100\%) fully on, the PWM signal must be adjusted from $0 \%$ duty cycle to $100 \%$ duty cycle. In contrast, to move a common servomotor over its full range, the PWM signal must be adjusted from a $1.2 \mathrm{~ms}$ pulse width $(6 \%$ duty cycle) to a $1.8 \mathrm{~ms}$ pulse width (9\% duty cycle). The output PWM conversion element of the Buttercup provides the flexibility to control a large number of different effectors.

\section{User Interface}

Although the Buttercup platform can operate independently of a PC, a PC is required to configure the device. The user interface for configuring the Buttercup platform is shown in Fig. 4. The graphical display, labeled C, gives the designer complete control over the sensor-to-effector response curve (black lines). In this figure, the preset cosine curve has been loaded. Each point on this curve can be dragged and dropped, allowing fine control over the system's response. Within this same display, the blue crosshairs provide the real-time operating point of the system. This feedback allows the user to quickly see how the system is responding and where adjustments need to be made. This real-time response is especially useful for determining the operating parameters of a sensor since the designer can see the real-time sensor output. 


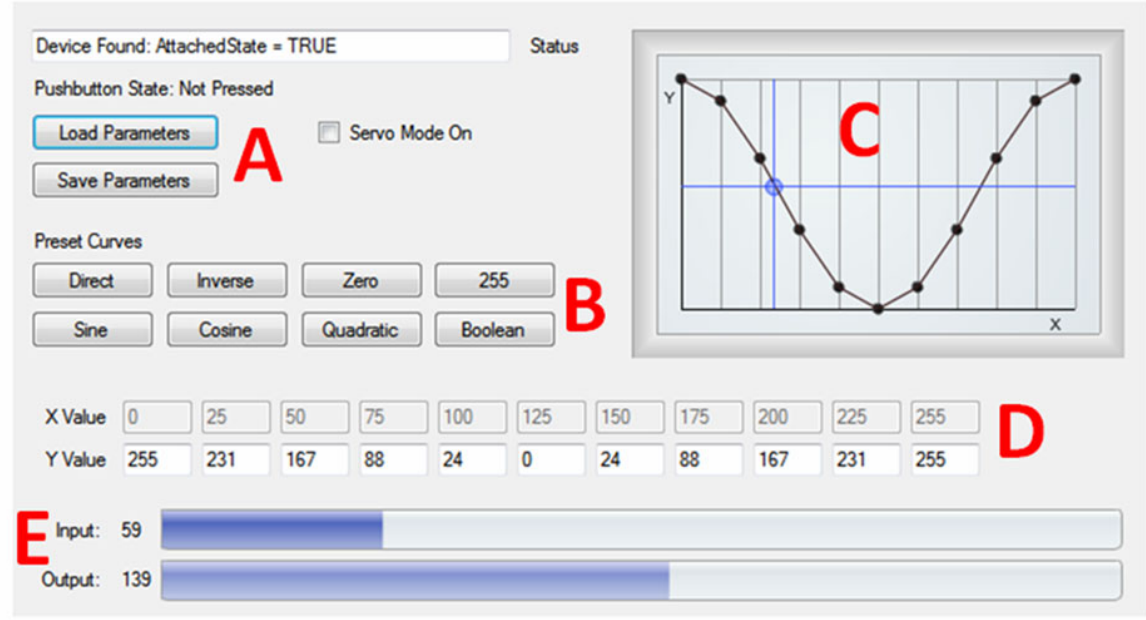

Fig. 4. Screenshot of the user interface: The large red letters are not part of the actual interface and are used only to highlight important aspects of the interface. A: Buttons used to load and save data to the Buttercup. B: Common curves can be loaded with these buttons. C: Graphical display of the sensor-to-effector response curve. Blue crosshairs provides real-time data of the current operating mode. D: Text boxes show numerical values of sensor-to-effector response curve. E: Real-time data of the sensor input signal and the effector output control signal.

The meter bars on the bottom, labeled E, provide real-time values of the sensor reading and the desired effector output. The Servo Mode On checkbox near label A allows the designer to select a PWM output that is compatible with a servo, or when unselected, typical PWM duty cycle control is used.

The Load Parameters button near label A loads the current response curve in volatile memory of the Buttercup controller. The Save Parameters button puts the response curve into non-volatile memory which is loaded on startup and allows independent operation.

This user interface and the low level USB communication was written in C\# with the exception of the response curve and meters bars, which were written in Flash.

\section{Conclusion}

Reducing the time between design iterations is critical to speeding up the prototyping process. Currently, there is no other embeddable system that provides a convenient method for rapidly prototyping interactive systems with a large range of accessories. Buttercup fills this need by providing an effective platform for prototyping devices that utilize sensors and effectors. The Buttercup platform gives designers complete control over their system's response. The parameters of the Buttercup platform are controlled with an intuitive user interface that allows the designer to adjust critical parameters of the interactive system. This enables the designer to quickly and effectively test new design concepts. Future work includes expanding on the types of 
sensors and effectors that Buttercup supports, adding support for multiple sensors and effectors, and increasing the functionality to include different types of control schemes.

\section{References}

1. Gill, S.: Six Challenges Facing User-oriented Industrial Design. The Design Journal 12 (2009)

2. Ahmed, S.M.Z.: A user-centred design and evaluation of IR interfaces. Journal of Librarianship and Information Science 38, 157-172 (2006)

3. Avrahami, D., Hudson, S.: Forming interactivity: a tool for rapid prototyping of physical interactive products. In: 4th Conference on Designing Interactive, pp. 141-146 (2002)

4. Carlson, J., Han, R., Lao, S., Narayan, C., Sanghani, S.: Rapid prototyping of mobile input devices using wireless sensor nodes. In: Proceedings of the Fifth IEEE Workshop on Mobile Computing Systems \& Applications, pp. 21-29 (2003)

5. Hartmann, B., Klemmer, S.R., Bernstein, M., Abdulla, L., Burr, B., Robinson-Mosher, A., Gee, J.: Reflective physical prototyping through integrated design, test, and analysis. In: Proceedings of the 19th Annual ACM Symposium on User Interface Software and Technology - UIST 2006, p. 299 (2006)

6. Akaoka, E., Ginn, T., Vertegaal, R.: DisplayObjects: Prototyping functional physical interfaces on 3D styrofoam, paper or cardboard models. In: Proceedings of the Fourth International Conference on Tangible Embedded and Embodied Interaction, p. 49 (2010)

7. Gill, S., Loudon, G., Woolley, A., Hare, J., Walker, D., Dix, A., Ellis, D.R.: Rapid development of tangible interactive appliances: achieving the fidelity/time balance. International Journal of Arts and Technology 1, 309 (2008)

8. Hudson, S.E., Mankoff, J.: Rapid construction of functioning physical interfaces from cardboard, thumbtacks, tin foil and masking tape. In: Proceedings of the 19th Annual ACM Symposium on User Interface Software and Technology, p. 298. ACM, New York (2006)

9. Greenberg, S., Fitchett, C.: Phidgets: easy development of physical interfaces through physical widgets. In: Proceedings of the 14th Annual ACM Symposium on User Interface Software and Technology, pp. 209-218. ACM, New York (2001)

10. Ballagas, R., Memon, F., Reiners, R., Borchers, J.: iStuff mobile: rapidly prototyping new mobile phone interfaces for ubiquitous computing. In: Proceedings of the SIGCHI Conference on Human Factors in Computing Systems - CHI 2007, p. 1107. Citeseer (2007) 